\title{
Outcome of retrograde ureteric stenting as a urinary drainage procedure in ureteric obstruction related to malignant lesions
}

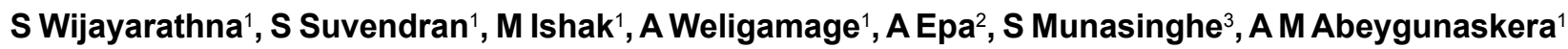

(Index words: obstructive uropathy, extrinsic malignant ureteric obstruction, Sri Lanka)

\begin{abstract}
Objectives We investigated the outcome of endoscopic retrograde ureteric stenting (RUS) in patients with ureteric obstruction related to malignant lesions.

Methods Data were prospectively collected from patients with ureteric obstruction related to malignant lesions treated at a single urology unit from 1 January 2011 to 30 April 2014. All patients had radiologically significant hydronephrosis by ultrasonography and CT scanning. First choice of urinary drainage was placing a retrograde ureteric stent cystoscopically. Outcome of patients who had stenting were recorded after following them until death or removal of stents.
\end{abstract}

Results Eighty two patients with ureteric obstruction related to malignant lesions had complete data. In 33 $(25 \%)$ patients, retrograde ureteric stenting was possible. Fifteen of them had recurrent tumour in the pelvis at the time of stenting and $13(87 \%)$ were dead within 3 months of stenting. Four of the five patients who did not have pelvic tumour recurrence were alive at the end of the study. All eight patients who underwent stenting before surgery as a prophylactic measure and three of the five patients who had retroperitoneal tumour mass and underwent RUS were alive. Stenting was not possible in 42 patients. Thirty eight of them had pelvic tumour recurrences and two were having retroperitoneal tumour masses. Only one did not have tumour in the pelvis.CT evidence of tumour recurrence in the pelvis (OR 12.7; 95\% Cl 1.3-117.6; $\mathrm{p}=0.026)$ and high serum creatinine (OR $4.3 ; 95 \% \mathrm{Cl}$ $1.6-11.7 ; p=0.004$ ) were associated with failure to ureteric stenting.

Conclusions Chances of successful RUS were low in patients with ureteric obstruction in the presence of tumour recurrences or elevated serum creatinine. Even if ureteric stenting was successful, their life expectancy was short.

Ceylon Medical Journal 2014; 59: 124-127

\section{Introduction}

Ureteric obstruction related to malignant lesions (UORM) commonly occurs due to recurrent malignancies in the pelvic cavity. However it could also be due to an advanced primary tumour in the pelvic cavity as well as peri-ureteric metastatic deposits in the retroperitoneal lymph nodes [1]. Rarely the obstructive uropathy may be related to the therapeutic measures given to the malignancy e.g. ureteric stricture due to radiotherapy or surgical scarring. Management of the obstructive uropathy of these patients poses a dilemma due to short survival duration, quality of life issues related to invasive procedures and risks of such interventions. Most of these patients have exhausted the primary modalities of treatment of their respective malignancies. This necessitates palliative care, as the sole option of therapy in a subgroup of patients with UORM. However a decision to provide palliative care only, is not easy due to patient and family expectations and emotional issues attached to the decision.

Once a decision is taken to drain the obstruction in patients with UORM, there are two main options endoscopic retrograde ureteric stenting (RUS) and percutaneous nephrostomy (PCN) [2]. A small minority of patients are suitable for resection of the tumour and reconstruction of the upper urinary tract. Each drainage procedure (RUS and PCN) has its own advantages and disadvantages. Need for regional or general anaesthesia, failure to insertion and stent related morbidity (irritative urinary symptoms, urinary sepsis, haematuria, encrustation and stone formation) are the disadvantages of stenting. Frequent tube dislodgement and having an external drainage system are the drawbacks of PCN. Both procedures relieve the obstruction only for a temporary period, as ureteric stents can be kept for a limited period of time. Hence the optimum management of ureteric obstruction related to malignant lesions is not well established.

A small number of patients with UORM who have not had any form of treatment for their malignancy are sent to the urologists for prophylactic ureteric stenting before the primary definitive surgery in order to identify and safeguard the ureters at the time of surgery and to improve the renal function preoperatively. This is a wellestablished, clear indication for RUS [3].

The pattern of causes for UORM differs according to the country. Common causes of UORM reported are

Departments of Urology' and Radiology ${ }^{2}$, Colombo South Teaching Hospital, Sri Lanka and ${ }^{3}$ Public Health Department, Norwich, UK.

Correspondence: SW, e-mail: <ksuranganuwan@yahoo.com>. Received 18 August 2014 and revised version accepted 06 October 2014. Competing interests: none declared. 
periureteric metastases of gastric cancer in Korea [2, 4] and carcinoma of prostate and bladder in Europe [5,6]. However, in Sri Lanka such malignancies are less common [7]. Hence the pattern and behaviour of obstruction and outcome of UORM and its treatment may be different in Sri Lanka [8]. Therefore it may not be possible to extrapolate recommendations regarding management guidelines of UORM based on other studies to Sri Lanka. It would be useful to investigate the characteristics of UORM and management outcome among patients in Sri Lanka in order to establish an evidence based protocol.

The aim of this study was to determine the outcome of RUS in patients with ureteric obstruction related to malignant lesions and to analyse the predictive factors for success in stent insertion and long-term survival.

\section{Methods}

Data were collected prospectively from patients with extrinsic ureteric obstruction related to a malignant lesion treated at urology unit, Colombo South Teaching Hospital from 1 January 2011 to 30 April 2014. All patients had radiologically significant hydronephrosis by ultrasono-graphy and CT scanning. Patients with carcinoma of prostate causing bladder outflow obstruction were excluded from the study while those with prostate cancer infiltrating the ureteric orifices and lower ureters causing obstruction were included. Several patients with known malignancies who were found to be having obstructive uropathy and were referred for intervention but were found to be having unrelated benign causes like stones and pelvi-ureteric junction obstruction were excluded from the study. Primary urothelial tumours of the ureter leading to intrinsic ureteric obstruction were also not included in the study.

First choice of urinary drainage was placing a retrograde ureteric stent cystoscopically as it was better tolerated by patients than a PCN $[9,10]$. Patients who had elevated serum creatinine over $3 \mathrm{mg} / \mathrm{dl}$ had initial haemodialysis prior to attempting RUS. Outcome of patients who had successful ureteric stenting were recorded after following them until death or removal of stents. In cases of ureteric stenting, a $6 \mathrm{Fr}, 26 \mathrm{~cm}$ long ureteric stent was passed over a guidewire using cystoscopic guidance under regional or general anaesthesia. PCN was performed using an $8 \mathrm{Fr}$ PCN tube by the radiologists under ultrasound guidance using local anaesthesia. Proper placement of tubes was confirmed by post-procedure X-Ray KUB.

Bivariate analysis was done using $2 \times 2$ tables to calculate the odds ratio in order to evaluate the variables for predicting the failure of stent insertion. A $p$ value less than 0.05 was considered statistically significant. Approval for the study was obtained from the Ethics Committee of Colombo South Teaching Hospital.

\section{Results}

There were 92 patients with UORM treated at Urology Unit of CSTH during the study period. Eighty two patients had complete data and were included in the analysis. There were 62 women $(\mathrm{F}: \mathrm{M}=2.5: 1)$. The mean age was 58 years (range 26-98 years). Sixty nine (84\%) patients were referred from the National Cancer Institute Maharagama.

Table 1 shows the distribution of the primary site of malignancy. The commonest causes were carcinoma of the cervix (48\%), endometrium (11\%) and rectosigmoid region of the colon (11\%). Fifty eight patients had been treated with previous radiotherapy and 50 had received chemotherapy. The obstruction was in the lower half of the ureter in $72(87.8 \%)$ patients.

\section{Table 1. Primary malignancy of the study population}

\begin{tabular}{lc}
\hline Site of malignancy & Number \\
\hline Cervix & 39 \\
Endometrium & 9 \\
Rectum/ sigmoid colon & 9 \\
Retropritoneal mass & 8 \\
Ovary & 6 \\
Prostate & 5 \\
Bladder & 3 \\
Sarcoma of pelvis & 2 \\
Vagina & 1 \\
Total & $\mathbf{8 2}$ \\
\hline
\end{tabular}

Table 2. Pattern of intervention and outcome of stenting

\begin{tabular}{cc}
\hline & Number \\
\hline Ureteric stenting successful & 33 \\
Pelvic tumour recurrence present & 15 \\
Pelvic tumour recurrence absent on CT & 5 \\
Retroperitoneal tumour mass & 5 \\
As prophylaxis before surgery & 8 \\
Ureteric stenting failed & 42 \\
Pelvic tumour recurrence present & 38 \\
Pelvic tumour recurrence absent on CT & 1 \\
Retroperitoneal tumour mass & 2 \\
As prophylaxis before surgery & 1 \\
Patient refusing stenting/ intervention & 1 \\
Open surgery done to relieve obstruction & 3 \\
Patients died awaiting ureteric stenting & 3 \\
Total & $\mathbf{8 2}$ \\
\hline
\end{tabular}


In $33(25 \%)$ patients RUS was possible. Fifteen of them had recurrent tumour in the pelvis at the time of stenting (CT and/or cystoscopic evidence) and 13 (87\%) were dead within 3 months of stenting (Table 2). Four of the five patients who did not have pelvic tumour recurrence were alive after six months. All eight patients who underwent RUS before definitive surgery were alive at the end of the study period. Three of the five patients who had retroperitoneal tumour mass and underwent RUS were alive at the end of study.

Stenting was not possible in 42 patients as ureteric orifice could not be identified due to the tumour tissue or the ureter did not allow the passage of a guidewire. Thirty eight of them had pelvic tumour recurrences, two were having retroperitoneal tumour masses and one was prior to primary definitive cancer surgery who had an extensive pelvic tumour compressing the bladder neck which made cystoscopic insertion impossible (Table 2). Only one did not have tumour recurrence.

Three patients died awaiting ureteric stenting, another three patients underwent open surgery to relieve the obstruction when the tumour mass was resectable (one uretero-sigmoidostomy, one transuretero-ureterostomy and one uretero-neocystostomy with a Boari flap). One patient refused to have any intervention. She had no pelvic tumour recurrence and was alive after one year with a stable serum creatinine level.

CT evidence of tumour recurrence in the pelvis (OR 12.7; 95\% CI 1.3-117.6; $\mathrm{p}=0.026$ ) and high serum creatinine prior to stenting (OR 4.3; 95\%CI 1.6-11.7; $\mathrm{p}=0.004)$ were associated with failure to stent (Tables 3 and 4).

\section{Discussion}

In the present study the distribution of malignancy causing the ureteric obstruction was different from that reported previously. Carcinoma of cervix which is the second commonest malignancy among women in Sri Lanka [7] was the commonest primary site of malignancy in this study. This resulted in a female preponderance in the study sample. The common causes of UORM described in other countries are periureteric metastases due to gastric cancer in Korea and carcinoma of prostate and bladder cancer in the UK and Europe [2,4-6]. These are also the common malignancies in the respective countries [11]. Colorectal cancer is the fourth commonest malignancy among Sri Lankan men and endometrial cancer is the eighth commonest malignancy among women in Sri Lanka [7]. Those were the second and third commonest causes of UORM in our study. Therefore the aetiology of UORM appears to be related to the pattern of malignancies in the native country.

There is no consensus on the optimum management of UORM as there are many variables in addition to the technical issues of RUS and PCN that affect the decision. These include certain subjective and emotional factors like dismal prognosis, quality of life issues and potential complications of invasive interventions in already seriously ill patients. Most of these patients do not have effective antineoplastic treatment options left.

Table 3. Association of failure to stent with presence of tumour recurrence

\begin{tabular}{lccc}
\hline & Failed stenting & Successful stenting & Total \\
\hline Tumour recurrence present & 38 & 15 & $\mathbf{5 3}$ \\
Tumour recurrence absent & 1 & 5 & $\mathbf{6}$ \\
Total & $\mathbf{3 9}$ & $\mathbf{2 0}$ & $\mathbf{5 9}$ \\
\hline
\end{tabular}

OR $12.7 ; 95 \%$ CI $1.3-117.6 ; p=0.026$

Table 4. Association of failure to stent with high serum creatinine level

\begin{tabular}{lccc}
\hline & Failed stenting & Successful stenting & Total \\
\hline Creatinine high & 32 & 14 & 46 \\
Creatinine low & 10 & 19 & 29 \\
Total & $\mathbf{4 2}$ & $\mathbf{3 3}$ & $\mathbf{7 5}$ \\
\hline
\end{tabular}

OR $4.3 ; 95 \%$ CI 1.6-11.7; $\mathrm{p}=0.004$ 
Most published series have a limited median survival of about 3-6 months [5,12].

The reported success rate of RUS is $37-47 \%[5,13]$. Our lower success rate of $25 \%$ may be due to high number of patients $(65 \%)$ with a tumour recurrences in the pelvis which is known to be factor associated with failure to ureteric stenting [5]. Our results suggest that patients with recurrent tumour in the pelvic cavity and high serum creatinine were less likely to have successful ureteric stenting. Even if RUS was successful in such patients the long-term outcome was dismal as only two patients $(2 / 13$, $15 \%$ ) were alive after three months. Therefore in patients with such risk factors it would be advisable to discuss options of PCN or even good palliative care rather than ureteric stenting that require multiple hospital visits and regional anaesthesia. Referral of such patients to urology units will not be meaningful. If such patients and families prefer relief of obstructive uropathy despite counselling, referral to an interventional radiologist for $\mathrm{PCN}$ would be more appropriate and fruitful.

Therefore if a patient who has had primary treatment for a pelvic malignancy develops evidence of UORM, he or she should have abdomino-pelvic imaging by ultrasonography and CT scanning in addition to renal function tests. If the imaging shows tumour recurrence in the pelvis and serum creatinine is high the attending clinician should have a discussion with the patient and family regarding the future course of action. If the patient and family opt to attempt relief of urinary tract obstruction, he/ she may be referred to an interventional radiologist for PCN. Those who do not have evidence of tumour recurrences in the pelvis should be referred to a urology unit for relief of obstruction by means of ureteric stenting.

In conclusion, management of UORM is a difficult clinical scenario in which the oncologist, interventional radiologist and urologist have to balance patient quality of life, renal preservation, limited effective options available to treat the recurrent malignancy and risks of invasive interventions in a situation of poor overall prognosis. Chances of successful RUS are very low in patients with ureteric obstruction by tumour recurrences in the pelvis after primary treatment. Even if RUS is successful, their life expectancy is short.

\section{References}

1. Russo P. Urologic emergencies in the cancer patient. Seminars in Oncology 2000; 27: 284-98.

2. Jeong IG, Han KS, Joung JY, Seo HK, Ching J. The outcome with ureteric stents for managing non-urological malignant ureteric obstruction. British Journal of Urology International 2007; 100: 1288-91.

3. Resnick MI, Kursh ED. Extrinsic obstruction of the ureter. In: Walsh PC, Retik AB, Vaughan ED, Wein AJ. Eds. Campbell's Urology. 7thedn. London: W.B. Saunders, 1998: 387-422.

4. Yu SH, Ryu JG, Jeong SH, et al. Predicting factors for stent failure-free survival in patients with a malignant ureteral obstruction managed with ureteral stents. Korean Journal of Urology 2013; 54: 316-21.

5. Shekarriz B, Shekarriz H, Upadhyay J, Banerjee M, Becker $\mathrm{H}$, Pontes JE, Wood DP. Outcome of palliative urinary diversion in the treatment of advanced malignancies. Cancer 1999; 85: 998-1003.

6. Jenkins CNJ, Marcus AJ. The value of antegrade stenting for lower ureteric obstruction. Journal of Royal Society of Medicine 1995; 88: 446-9.

7. Cancer Incidence Data Sri Lanka 2006, Cancer Registry National Cancer Control Programme Sri Lanka, 2012.

8. Samarasinghe UC, Perera ND. The value of percutaneous nephrostomy; Sri Lankan experience. Ceylon Medical Journal 1998; 43: 92-5.

9. Izumi K, Mizokami A, Maeda Y, Koh E, Namiki M. Current outcome of patients with ureteral stents for the management of malignant ureteral obstruction. Journal of Urology 2011; 185: 556-61.

10. Rosevear HM, Kim SP, Wenzler DL, Faerber GJ, Roberts WW, Wolf JS Jr. Retrograde ureteral stents for extrinsic ureteral obstruction: nine years' experience at University of Michigan. Urology 2007; 70: 846-50.

11. Ferlay J, Shin HR, Bray F, et al. GLOBOCAN 2008, Cancer incidence and mortality worldwide: IARC Cancer Base No. 10. Lyon, France: International Agency for Research on Cancer; 2010.

12. Kanou T, Fujiyama C, Nishimura K, Tokuda Y, Uozumi J, Masaki Z. Management of extrinsic malignant ureteral obstruction with urinary diversion. International Journal of Urology 2007; 14: 689-92.

13. Huebner WA, Plas EG, Porpaczy P. Hydronephrosis in malignant tumours: rational and efficiency of endourological diversion. European Journal of Oncology 1993; 189: $27-32$. 